\title{
An SIR Epidemic Model with Time Delay and General Nonlinear Incidence Rate
}

\author{
Mingming Li and Xianning Liu \\ Key Laboratory of Eco-Environments in Three Gorges Reservoir Region (Ministry of Education), \\ School of Mathematics and Statistics, Southwest University, Chongqing 400715, China \\ Correspondence should be addressed to Xianning Liu; liuxn@swu.edu.cn
}

Received 26 December 2013; Accepted 6 January 2014; Published 20 February 2014

Academic Editor: Weiming Wang

Copyright (C) $2014 \mathrm{M}$. Li and X. Liu. This is an open access article distributed under the Creative Commons Attribution License, which permits unrestricted use, distribution, and reproduction in any medium, provided the original work is properly cited.

\begin{abstract}
An SIR epidemic model with nonlinear incidence rate and time delay is investigated. The disease transmission function and the rate that infected individuals recovered from the infected compartment are assumed to be governed by general functions $F(S, I)$ and $G(I)$, respectively. By constructing Lyapunov functionals and using the Lyapunov-LaSalle invariance principle, the global asymptotic stability of the disease-free equilibrium and the endemic equilibrium is obtained. It is shown that the global properties of the system depend on both the properties of these general functions and the basic reproductive number $R_{0}$.
\end{abstract}

\section{Introduction}

The mechanism of transmission is usually qualitatively known for most diseases from epidemiological point of view. For modeling the spread process of infectious diseases mathematically and quantitatively, many classical epidemic models have been proposed and studied, such as SIR, SIS, SEIR, and SIRS models. Recently, considerable attention have been paid to study the dynamics of epidemic models with epidemiological meaningful time delays.

The fundamental assumption in epidemic models is that the population can be divided into distinct groups. The most common groups are the susceptible $(S)$ which contains individuals that may be infected by the disease; the infected (I) which contains individuals that are already infected and can spread the disease to susceptible individuals; the removed $(R)$ which contains individuals that have the immunity and cannot be infected. Therefore such models are referred to SIR models. The simplest forms of these models are ordinary differential equations (ODEs).

It is well known that the disease transmission progress plays an important role in the epidemic dynamics; that is, applying different incidence rates can potentially change the behaviors of the system. In many epidemic models, following incidence functions with delay or without delay are widely used in different epidemiological backgrounds.
(1) The bilinear incidence rate $\beta S I$ (e.g., [1-8]), where $\beta$ is the average number of contacts per infected individual per day.

(2) The standard incidence rate $\beta S I / N$ ([9-12]), where $N=S+I+R$.

(3) The Holling type incidence rate of the form $\beta S I /(1+$ $\left.\alpha_{1} S\right)([13-15])$, where $\alpha_{1}$ is a positive constant.

(4) The saturated incidence rate of the form $\beta S I /\left(1+\alpha_{2} I\right)$ ([16-21]), where $\alpha_{2}$ is a positive constant.

(5) The saturated incidence rate of the form $\beta S I /\left(1+\alpha_{1} S+\right.$ $\left.\alpha_{2} I\right)([22-25])$, where $\alpha_{1}, \alpha_{2}$ is a positive constant.

The bilinear incidence rate in (1) is based on the law of mass action, which is more appropriate for communicable diseases, such as influenza, but not suitable for sexually transmitted diseases. It has been pointed out that standard incidence rate in (2) may be a good approximation when the number of available partners is large enough and everybody could not make more contacts than that is practically feasible. In fact, the infection probability per contact is likely influenced by the number of infective individuals, because more infective individuals can increase the infection risk.

In the incidence rates in (3) and (4), $\beta S I$ measures the infection force of the disease and $1 /\left(1+\alpha_{1} S\right), 1 /\left(1+\alpha_{2} I\right)$ measure the inhibition effect from the behavioral changes of 
the susceptible individuals when their number increases or from the crowding effect of the infective individuals. In these incidence rates, the number of effective contacts between infectived and susceptible individuals may saturate at high infective levels. These incidence rates seem more reasonable than the bilinear incidence rate $\beta S I$, because they include the behavioral changes of susceptible individuals and crowding effect of the infective individuals which prevent the unboundedness of the contact rate by choosing suitable parameters.

Obviously, the incidence rate in (5) includes the former three incidence rates: the bilinear incidence rate $\beta S I$ (when $\left.\alpha_{1}=0, \alpha_{2}=0\right)$, the Holling type incidence rate $\beta S I /\left(1+\alpha_{1} S\right)$ (when $\left.\alpha_{2}=0\right)$, and the saturated incidence rate $\beta S I /\left(1+\alpha_{2} I\right)$ (when $\alpha_{1}=0$ ).

The incidence rate can also be modeled by many other kinds of more general functions. It is interesting that whether the functional form of the incidence rate can change the epidemic dynamics or not. Korobeinikov studied the global properties for epidemiological models with various nonlinear incidence rates, such as $f(s) g(i)$ in [26], $f(s, i)$ in [27-29]. By constructing Lyapunov functions, $[27,28]$ established the global stability for ordinary differential equations models of epidemiological dynamics with nonlinear incidence rate $f(s, i)$ under some conditions.

These models have not included time delays, which are usually used to model the fact that an individual may not be infectious until some time after becoming infected. In the context of epidemiology, delays can be caused by a variety of factors. The most common reasons for a delay are (i) the latency of the infection in a vector and (ii) the latency of the infection in an infected host [30]. In these cases, some time should elapse before the level of infection in the infected host or the vector reaching a sufficiently high level to transmit the infection further.

Motivated by all the above, we present a model described by delay differential equations (DDEs) with two general nonlinear terms as follows:

$$
\begin{gathered}
\frac{d S(t)}{d t}=\lambda-\mu S(t)-F(S(t), I(t)), \\
\frac{d I(t)}{d t}=e^{-\mu \tau} F(S(t-\tau), I(t-\tau))-(\mu+\alpha) I(t)-G(I(t)), \\
\frac{d R(t)}{d t}=G(I(t))-\mu R(t),
\end{gathered}
$$

where $S(t), I(t)$, and $R(t)$, as mentioned above, represent the population of the susceptible, the infected, and the removed at time $t$, respectively. The parameters in the equations are explained as below. The positive $\lambda$ is the recruitment rate of the population, $\mu$ is the natural death rate of the population, $\alpha$ is the death rate due to disease, all $\tau \geq 0$ is the latent period. The term $0 \leq e^{-\mu \tau}<1$ represents the survival rate of population and the time they take to become infectious is $\tau$. We assume that the force of infection at any time $t$ is given by the general function $F(S(t), I(t))$, and the recovered infected individuals at any time $t$ is given by the function $G(I(t))$.
Since $R(t)$ does not appear in equations for $d S(t) / d t$ and $d I(t) / d t$, it is sufficient to analyze the behaviors of solutions of (1) by the following system of DDEs:

$$
\begin{gathered}
\frac{d S(t)}{d t}=\lambda-\mu S(t)-F(S(t), I(t)), \\
\frac{d I(t)}{d t}=e^{-\mu \tau} F(S(t-\tau), I(t-\tau))-(\mu+\alpha) I(t)-G(I(t)) .
\end{gathered}
$$

The initial conditions for system (2) take the form

$$
\begin{gathered}
S(\theta)=\phi_{1}(\theta), \quad I(\theta)=\phi_{2}(\theta), \\
\phi_{1}(\theta) \geq 0, \quad \phi_{2}(\theta) \geq 0, \quad \theta \in[-\tau, 0], \\
\phi_{1}(0)>0, \quad \phi_{2}(0)>0,
\end{gathered}
$$

where $\phi=\left(\phi_{1}(\theta), \phi_{2}(\theta)\right) \in C^{+} \times C^{+}$. Here $C$ denotes the Banach space $C=C([-\tau, 0], \mathbb{R})$ of continuous functions mapping the interval $[-\tau, 0]$ into $\mathbb{R}$, equipped with the supremum norm. The nonnegative cone of $C$ is defined as $C^{+}=C\left([-\tau, 0], \mathbb{R}^{+}\right)$.

The organization of this paper is as follows. In Section 2, we study the existence of a positive equilibrium. In Section 3, we show that the global asymptotic stability of the diseasefree equilibrium and the endemic equilibrium of model (2) depend only on the basic reproductive number under some hypotheses. A brief discussion is given in the last section to conclude this paper.

\section{The Existence of Positive Equilibrium}

In this section, we prove the existence of a positive equilibrium. We assume that $F(S, I)$ and $G(I)$ are always positive, continuously differentiable, and monotonically increasing for all $S>0$ and $I>0$. That is, they satisfy the following conditions:

(H1) $F(S, I)>0, F_{S}^{\prime}(S, I)>0, F_{I}^{\prime}(S, I)>0$ for $S>0$ and $I>0$.

(H2) $F(S, 0)=F(0, I)=0, F_{S}^{\prime}(S, 0)=0, F_{I}^{\prime}(S, 0)>0$ for $S>0$ and $I>0$.

(H3) $G(0)=0 . G^{\prime}(I)>0$ for $I \geq 0$.

Global behaviors of system (2) may depend on the basic reproduction number $R_{0}$, which is the average number of secondary cases produced by a single infective individual introduced into an entirely susceptible population. The basic reproductive number for system (2) can be computed as

$$
R_{0}=\frac{e^{-\mu \tau} F_{I}^{\prime}\left(S_{0}, 0\right)}{\mu+\alpha+G^{\prime}(0)},
$$

where $S_{0}=\lambda / \mu$. Usually, $R_{0}<1$ implies that the number of infected individuals will tend to zero and $R_{0}>1$ implies that the number will increase.

The epidemiologically natural condition $F(S, 0)=0$ ensures that system (2) always has a disease-free equilibrium $E_{0}=\left(S_{0}, 0\right)$. And it may also admit an endemic equilibrium 
$E^{*}=\left(S^{*}, I^{*}\right)$ which depends on $R_{0}$. And $S^{*}, I^{*}$ satisfy the following equations:

$$
\begin{gathered}
\lambda-\mu S^{*}=F\left(S^{*}, I^{*}\right), \\
F\left(S^{*}, I^{*}\right)=e^{\mu \tau}\left[(\mu+\alpha) I^{*}+G\left(I^{*}\right)\right] .
\end{gathered}
$$

We will show that under certain epidemiologically reasonable conditions, the existence of the positive equilibrium state $E^{*}$ is ensured. We have the following theorem.

Theorem 1. Assume that $F(S, I)$ satisfies (H1) and (H2), and $G(I)$ satisfies (H3). Then system (2) has a positive equilibrium state $E^{*}=\left(S^{*}, I^{*}\right)$ if $R_{0}>1$.

Proof. Let the right-hand sides of the three equations in system (2) equal zero; we have that

$$
\lambda-\mu S=F(S, I)=e^{\mu \tau}[(\mu+\alpha) I+G(I)] .
$$

Substituting the expression of $S$ by $I$, we obtain the following equation for $I$ :

$$
\begin{aligned}
H(I)= & F\left(\frac{\lambda-e^{\mu \tau}[(\mu+\alpha) I+G(I)]}{\mu}, I\right) \\
& -e^{\mu \tau}[(\mu+\alpha) I+G(I)] .
\end{aligned}
$$

It is obvious that $H(0)=0$, and we can compute that there exists a positive $I_{0}$ such that $\lambda=e^{\mu \tau}\left[(\mu+\alpha) I_{0}+G\left(I_{0}\right)\right]$. Hence

$$
H\left(I_{0}\right)=F\left(0, I_{0}\right)-\lambda=-\lambda<0 .
$$

And when $I \geq 0$, since $H(I)$ is continuously differentiable, we have

$$
\begin{aligned}
H^{\prime}(0)= & \lim _{I \rightarrow 0^{+}} \frac{H(I)-H(0)}{I-0} \\
= & F_{I}^{\prime}\left(S_{0}, 0\right)-e^{\mu \tau}\left[\mu+\alpha+G^{\prime}(0)\right] F_{S}^{\prime}\left(S_{0}, 0\right) \\
& -e^{\mu \tau}\left[\mu+\alpha+G^{\prime}(0)\right] \\
= & F_{I}^{\prime}\left(S_{0}, 0\right)-e^{\mu \tau}\left[\mu+\alpha+G^{\prime}(0)\right] \\
= & e^{\mu \tau}\left[\mu+\alpha+G^{\prime}(0)\right]\left(R_{0}-1\right) .
\end{aligned}
$$

Thus, $R_{0}>1$ ensures that $H^{\prime}(0)>0$. And $H(I)$ is continuous on $\left[0, I_{0}\right]$; then there exists some $I^{*} \in\left(0, I_{0}\right)$ such that $H\left(I^{*}\right)=0$. Since $G(I)$ is strictly monotonically increasing, we have $e^{\mu \tau}\left[(\mu+\alpha) I^{*}+G\left(I^{*}\right)\right]<e^{\mu \tau}\left[(\mu+\alpha) I_{0}+G\left(I_{0}\right)\right]$. Therefore $S^{*}=\left(\lambda-e^{\mu \tau}\left[(\mu+\alpha) I^{*}+G\left(I^{*}\right)\right]\right) / \mu>0$, and we have proved the existence of the endemic equilibrium $E^{*}=\left(S^{*}, I^{*}\right)$ for system (2) under condition $R_{0}>1$. This completes the proof.

\section{Global Dynamics of the Model}

In this section, we will analyze the global dynamics of system (2) and show the global asymptotic stability of the disease-free equilibrium and the endemic equilibrium.
3.1. Stability of the Disease-Free Equilibrium. In this subsection, we will study the global stability of the disease-free equilibrium $E_{0}=\left(S_{0}, 0\right)$ of system (2). We propose the following conditions:

(H4) $F_{I}^{\prime}(S, 0)$ is increasing with respect to $S>0$.

(H5) $F(S, I) \leq I \cdot(\partial F(S, 0) / \partial I)$ with respect to $I>0$.

(H6) $G^{\prime}(0) \leq G(I) / I$ with respect to $I>0$.

By (H4), the following inequalities hold true:

$$
\begin{gathered}
\frac{F_{I}^{\prime}\left(S_{0}, 0\right)}{F_{I}^{\prime}(S, 0)}>1 \quad \text { for } S \in\left(0, S_{0}\right), \\
\frac{F_{I}^{\prime}\left(S_{0}, 0\right)}{F_{I}^{\prime}(S, 0)}<1 \quad \text { for } S>S_{0} .
\end{gathered}
$$

Under these conditions, we have the following theorems.

Theorem 2. Suppose that conditions $(\mathrm{H} 1)-(\mathrm{H} 3)$ are satisfied. Then the disease-free equilibrium $E_{0}=\left(S_{0}, 0\right)$ of system (2) is locally asymptotically stable for any $\tau>0$ if $R_{0}<1 ; E_{0}=$ $\left(S_{0}, 0\right)$ is unstable if $R_{0}>1$.

Proof. The characteristic equation of system (2) at $E_{0}=$ $\left(S_{0}, 0\right)$ is

$$
(\lambda+u)\left(\lambda-e^{-\tau(\lambda+\mu)} F_{I}^{\prime}\left(S_{0}, 0\right)+\mu+\alpha+G^{\prime}(0)\right)=0 .
$$

It has a negative real root $\lambda_{1}=-\mu$. Moreover, it has a root of

$$
\lambda-e^{-\tau(\lambda+\mu)} F_{I}^{\prime}\left(S_{0}, 0\right)+\mu+\alpha+G^{\prime}(0)=0 .
$$

In (12), if $\tau=0, R_{0}<1$ becomes $R_{01}=F_{I}^{\prime}\left(S_{0}, 0\right) /[\mu+\alpha+$ $\left.G^{\prime}(0)\right]<1$; one can see that $\lambda_{2}=\left[\mu+\alpha+G^{\prime}(0)\right]\left(R_{01}-1\right)<0$. Hence the $E_{0}=\left(S_{0}, 0\right)$ is locally asymptotically stable. In (12), if $\tau>0, x>0, y \neq 0, \lambda=x+i \cdot y$ is a root of (12), then we have

$$
\begin{gathered}
x+\mu+\alpha+G^{\prime}(0)=e^{-\tau(x+\mu)} F_{I}^{\prime}\left(S_{0}, 0\right) \cos (y \tau), \\
y=-e^{-\tau(x+\mu)} F_{I}^{\prime}\left(S_{0}, 0\right) \sin (y \tau) .
\end{gathered}
$$

Further we have

$$
\begin{aligned}
{\left[x+\mu+\alpha+G^{\prime}(0)\right]^{2}+y^{2} } & =\left[e^{-\tau(x+\mu)} F_{I}^{\prime}\left(S_{0}, 0\right)\right]^{2} \\
& \leq\left[e^{-\mu \tau} F_{I}^{\prime}\left(S_{0}, 0\right)\right]^{2},
\end{aligned}
$$

which is a contradiction. Hence the $E_{0}=\left(S_{0}, 0\right)$ is locally asymptotically stable.

If $R_{0}>1$, let $h(\lambda)=\lambda-e^{-\tau(\lambda+\mu)} F_{I}^{\prime}\left(S_{0}, 0\right)+\mu+\alpha+G^{\prime}(0)$; then we have

$$
\begin{gathered}
h(0)=\left[\mu+\alpha+G^{\prime}(0)\right]\left(1-R_{0}\right)<0, \\
\lim _{\lambda \rightarrow+\infty} h(\lambda)=+\infty .
\end{gathered}
$$

And when $\lambda \geq 0$, since $h(\lambda)$ is continuously, then $h(\lambda)=0$ has at least one positive root. Hence $E_{0}=\left(S_{0}, 0\right)$ is unstable. This completes the proof. 
Theorem 3. Suppose that conditions (H1)-(H6) are satisfied. Then the disease-free equilibrium $E_{0}=\left(S_{0}, 0\right)$ of system (2) is globally asymptotically stable for any $\tau>0$ if $R_{0} \leq 1$.

Proof. Define a Lyapunov functional

$$
V_{1}(t)=U_{1}(t)+U_{2}(t),
$$

where

$$
\begin{gathered}
U_{1}(t)=S(t)-S_{0}-\int_{S_{0}}^{S(t)} \lim _{I \rightarrow 0^{+}} \frac{F\left(S_{0}, I(t)\right)}{F(\theta, I(t))} d \theta+e^{\mu \tau} I(t), \\
U_{2}(t)=\int_{0}^{\tau} F(S(t-\eta), I(t-\eta)) d \eta .
\end{gathered}
$$

By (H1)-(H6), it is obvious that $V_{1}$ is defined and continuously differentiable for all $S(t), I(t)>0$, and $V_{1}=0$ at $E_{0}=$ $\left(S_{0}, 0\right)$. The system $(2)$ at $E_{0}=\left(S_{0}, 0\right)$ has $\lambda=\mu S_{0}$. The time derivative of $U_{1}$ along the solutions of system (2) is given by

$$
\begin{aligned}
\frac{d U_{1}(t)}{d t} & S^{\prime}(t)-\lim _{I \rightarrow 0^{+}} \frac{F\left(S_{0}, I(t)\right)}{F(S(t), I(t))} \cdot S^{\prime}(t)+e^{\mu \tau} I^{\prime}(t) \\
= & \left(1-\lim _{I \rightarrow 0^{+}} \frac{F\left(S_{0}, I(t)\right)}{F(S(t), I(t))}\right)[\lambda-\mu S(t)-F(S(t), I(t))] \\
& +F(S(t-\tau), I(t-\tau))-e^{\mu \tau}[(\mu+\alpha) I(t)+G(I(t))] \\
= & \mu S(t)\left(\frac{S_{0}}{S(t)}-1\right)\left(1-\lim _{I \rightarrow 0^{+}} \frac{F\left(S_{0}, I(t)\right)}{F(S(t), I(t))}\right) \\
& -F(S(t), I(t))+F(S(t), I(t)) \cdot \lim _{I \rightarrow 0^{+}} \frac{F\left(S_{0}, I(t)\right)}{F(t), I(t))} \\
& +F(S(t-\tau), I(t-\tau))-e^{\mu \tau}[(\mu+\alpha) I(t)+G(I(t))] .
\end{aligned}
$$

Further, we have

$$
\begin{aligned}
\frac{d U_{2}(t)}{d t} & =\frac{d}{d t} \int_{0}^{\tau} F(S(t-\eta), I(t-\eta)) d \eta \\
& =F(S(t), I(t))-F(S(t-\tau), I(t-\tau)) .
\end{aligned}
$$

Thus

$$
\begin{aligned}
\frac{d V_{1}(t)}{d t}= & \frac{d U_{1}(t)}{d t}+\frac{d U_{2}(t)}{d t} \\
= & \mu S(t)\left(\frac{S_{0}}{S(t)}-1\right)\left(1-\lim _{I \rightarrow 0^{+}} \frac{F\left(S_{0}, I(t)\right)}{F(S(t), I(t))}\right) \\
& +F(S(t), I(t)) \cdot \lim _{I \rightarrow 0^{+}} \frac{F\left(S_{0}, I(t)\right)}{F(S(t), I(t))} \\
& -e^{\mu \tau}[(\mu+\alpha) I(t)+G(I(t))] .
\end{aligned}
$$

By (10), we have

$$
\begin{aligned}
& \left(\frac{S_{0}}{S(t)}-1\right)\left(1-\lim _{I \rightarrow 0^{+}} \frac{F\left(S_{0}, I(t)\right)}{F(S(t), I(t))}\right) \\
& \quad=\left(\frac{S_{0}}{S(t)}-1\right)\left(1-\frac{F_{I}^{\prime}\left(S_{0}, 0\right)}{F_{I}^{\prime}(S(t), 0)}\right) \leq 0 .
\end{aligned}
$$

Note that $F_{I}^{\prime}\left(S_{0}, 0\right) / F_{I}^{\prime}(S, 0) \neq 1$, for $S \neq S_{0}, S>0$, and by $F_{I}^{\prime}(S, 0)>0$ and (H4), the equality in (21) holds if and only if $S=S_{0}$. Furthermore, (H5) and (H6) imply that

$$
\begin{aligned}
F(S(t), I(t)) \cdot \lim _{I \rightarrow 0^{+}} \frac{F\left(S_{0}, I(t)\right)}{F(S(t), I(t))} \\
\quad-e^{\mu \tau}[(\mu+\alpha) I(t)+G(I(t))] \\
=F(S(t), I(t)) \cdot \frac{F_{I}^{\prime}\left(S_{0}, 0\right)}{F_{I}^{\prime}(S(t), 0)} \\
\quad-e^{\mu \tau}[(\mu+\alpha) I(t)+G(I(t))] \\
\leq I(t) \cdot\left[F_{I}^{\prime}\left(S_{0}, 0\right)-e^{\mu \tau}\left(\mu+\alpha+G^{\prime}(0)\right)\right] \\
=e^{\mu \tau}\left(\mu+\alpha+G^{\prime}(0)\right) I(t)\left(R_{0}-1\right) .
\end{aligned}
$$

Therefore, $R_{0} \leq 1$ ensures that $d V_{1} / d t \leq 0$ for all $S(t) \geq 0$, $I(t) \geq 0$, where $d V_{1} / d t=0$ holds only for $S=S_{0}$. It is easy to verify that the disease-free equilibrium $E_{0}$ is the only fixed point of the systems on the plane $S=S_{0}$ and hence it is easy to show that the largest invariant set in $\left\{(S(t), I(t)) \mid d V_{1} / d t=\right.$ $0\}$ is the singleton $\left\{E_{0}\right\}$. By the Lyapunov-LaSalle asymptotic stability theorem in [31], $E_{0}$ is globally asymptotically stable for any $\tau>0$. This completes the proof.

3.2. Global Stability of the Endemic Equilibrium. In this subsection, we will study the global stability of the endemic equilibrium $E^{*}=\left(S^{*}, I^{*}\right)$ of system (2) by the Lyapunov direct method. We propose the following hypotheses:

(H7) $I / I^{*} \leq F(S, I) / F\left(S, I^{*}\right)$ for $I \in\left(0, I^{*}\right), F(S, I) /$ $F\left(S, I^{*}\right) \leq I / I^{*}$ for $I \geq I^{*}$.

(H8) $G(I) / G\left(I^{*}\right) \leq I / I^{*}$ for $I \in\left(0, I^{*}\right), I / I^{*} \leq G(I) / G\left(I^{*}\right)$ for $I \geq I^{*}$.

Based on these, we have the following theorem.

Theorem 4. Suppose that conditions (H1)-(H3) and (H7)(H8) are satisfied. Then the endemic equilibrium $E^{*}=\left(S^{*}, I^{*}\right)$ of system (2) is globally asymptotically stable for any $\tau>0$ if $R_{0}>1$.

Proof. Define a Lyapunov functional

$$
V_{2}(t)=W_{1}(t)+W_{2}(t),
$$

where

$$
\begin{aligned}
W_{1}(t)= & S(t)-S^{*}-\int_{S^{*}}^{S(t)} \frac{F\left(S^{*}, I^{*}\right)}{F\left(\varphi, I^{*}\right)} d \varphi \\
& +e^{\mu \tau}\left(I(t)-I^{*}-I^{*} \ln \frac{I(t)}{I^{*}}\right) .
\end{aligned}
$$




$$
\begin{array}{cc}
W_{2}(t)=F\left(S^{*}, I^{*}\right) \int_{0}^{\tau}\left(\frac{F(S(t-\xi), I(t-\xi))}{F\left(S^{*}, I^{*}\right)}-1\right. & =F(S(t), I(t))-F(S(t-\tau), I(t-\tau)) \\
\left.-\ln \frac{F(S(t-\xi), I(t-\xi))}{F\left(S^{*}, I^{*}\right)}\right) d \xi & +F\left(S^{*}, I^{*}\right) \ln \frac{F(S(t-\tau), I(t-\tau))}{F(S(t), I(t))}
\end{array}
$$

By $(\mathrm{H} 1)-(\mathrm{H} 6), V_{2}(t)=W_{1}(t)+W_{2}(t)$ is defined and continuously differentiable for all $S(t), I(t)>0$. And $V_{2}(0)=$ 0 at $E^{*}=\left(S^{*}, I^{*}\right)$. At $E^{*}=\left(S^{*}, I^{*}\right)$, system (2) has

$$
\begin{gathered}
\lambda-\mu S^{*}=F\left(S^{*}, I^{*}\right), \\
F\left(S^{*}, I^{*}\right)=e^{\mu \tau}\left[(\mu+\alpha) I^{*}+G\left(I^{*}\right)\right] .
\end{gathered}
$$

The time derivative of $W_{1}$ along the solutions of system (2) is given by

$$
\left.\begin{array}{rl}
\frac{d W_{1}(t)}{d t} & S^{\prime}(t)-\frac{F\left(S^{*}, I^{*}\right)}{F\left(S(t), I^{*}\right)} S^{\prime}(t)+e^{\mu \tau} I^{\prime}(t)\left(1-\frac{I^{*}}{I(t)}\right) \\
= & \left(1-\frac{F\left(S^{*}, I^{*}\right)}{F\left(S(t), I^{*}\right)}\right)\left[\mu S^{*}-\mu S(t)+F\left(S^{*}, I^{*}\right)\right.
\end{array}\right]
$$

Further, we have

$$
\begin{aligned}
& \frac{d W_{2}(t)}{d t} \\
& =F\left(S^{*}, I^{*}\right) \cdot \frac{d}{d t} \int_{0}^{\tau}\left(\frac{F(S(t-\xi), I(t-\xi))}{F\left(S^{*}, I^{*}\right)}-1\right. \\
& \left.\quad-\ln \frac{F(S(t-\xi), I(t-\xi))}{F\left(S^{*}, I^{*}\right)}\right) d \xi
\end{aligned}
$$

Then we have

$$
\begin{aligned}
& \frac{d V_{2}(t)}{d t}=\frac{d W_{1}(t)}{d t}+\frac{d W_{2}(t)}{d t} \\
& =\mu S^{*}\left(1-\frac{F\left(S^{*}, I^{*}\right)}{F\left(S(t), I^{*}\right)}\right)\left(1-\frac{S(t)}{S^{*}}\right) \\
& +F\left(S^{*}, I^{*}\right)\left(1-\frac{F\left(S^{*}, I^{*}\right)}{F\left(S(t), I^{*}\right)}+\frac{F(S(t), I(t))}{F\left(S(t), I^{*}\right)}\right) \\
& +F\left(S^{*}, I^{*}\right)\left(1-\frac{I(t)}{I^{*}}-\frac{I^{*}}{I(t)}\right. \\
& \frac{F(S(t-\tau), I(t-\tau))}{F\left(S^{*}, I^{*}\right)} \\
& \left.+\ln \frac{F(S(t-\tau), I(t-\tau))}{F(S(t), I(t))}\right) \\
& +e^{\mu \tau} G\left(I^{*}\right)\left[\frac{I(t)}{I^{*}}-1-\frac{G(I(t))}{G\left(I^{*}\right)}\right. \\
& \left.+\frac{G(I(t))}{G\left(I^{*}\right)} \cdot \frac{I^{*}}{I(t)}\right] \\
& =\mu S^{*}\left(1-\frac{F\left(S^{*}, I^{*}\right)}{F\left(S(t), I^{*}\right)}\right)\left(1-\frac{S(t)}{S^{*}}\right) \\
& +F\left(S^{*}, I^{*}\right)\left(1-\frac{F\left(S^{*}, I^{*}\right)}{F\left(S(t), I^{*}\right)}+\ln \frac{F\left(S^{*}, I^{*}\right)}{F\left(S(t), I^{*}\right)}\right) \\
& +F\left(S^{*}, I^{*}\right)\left(1-\frac{I^{*}}{I(t)} \cdot \frac{F(S(t-\tau), I(t-\tau))}{F\left(S^{*}, I^{*}\right)}\right. \\
& \left.+\ln \frac{I^{*}}{I(t)} \cdot \frac{F(S(t-\tau), I(t-\tau))}{F\left(S^{*}, I^{*}\right)}\right) \\
& +F\left(S^{*}, I^{*}\right)\left(1-\frac{I(t)}{I^{*}} \cdot \frac{F\left(S(t), I^{*}\right)}{F(S(t), I(t))}\right. \\
& \left.+\ln \frac{I(t)}{I^{*}} \cdot \frac{F\left(S(t), I^{*}\right)}{F(S(t), I(t))}\right) \\
& +F\left(S^{*}, I^{*}\right)\left(\frac{I(t)}{I^{*}}-\frac{F(S(t), I(t))}{F\left(S(t), I^{*}\right)}\right) \\
& \times\left(\frac{F\left(S(t), I^{*}\right)}{F(S(t), I(t))}-1\right) \\
& +e^{\mu \tau} G\left(I^{*}\right)\left(\frac{G(I(t))}{G\left(I^{*}\right)}-\frac{I(t)}{I^{*}}\right)\left(\frac{I^{*}}{I(t)}-1\right) .
\end{aligned}
$$


The function $F(S, I)$ is monotonically increasing for any $S>$ 0 ; hence the following inequality holds:

$$
\left(1-\frac{F\left(S^{*}, I^{*}\right)}{F\left(S(t), I^{*}\right)}\right)\left(1-\frac{S(t)}{S^{*}}\right) \leq 0 .
$$

And by the properties of the function $g(x)=1-x+\ln x$, $(x>0)$, we note that $g(x)$ has its global maximum $g(1)=0$. Hence $g(x) \leq 0$ when $x>0$ and the following inequalities hold true:

$$
\begin{gathered}
1-\frac{F\left(S^{*}, I^{*}\right)}{F\left(S(t), I^{*}\right)}+\ln \frac{F\left(S^{*}, I^{*}\right)}{F\left(S(t), I^{*}\right)} \leq 0, \\
1-\frac{I^{*}}{I(t)} \cdot \frac{F(S(t-\tau), I(t-\tau))}{F\left(S^{*}, I^{*}\right)} \\
+\ln \frac{I^{*}}{I(t)} \cdot \frac{F(S(t-\tau), I(t-\tau))}{F\left(S^{*}, I^{*}\right)} \leq 0, \\
1-\frac{I(t)}{I^{*}} \cdot \frac{F\left(S(t), I^{*}\right)}{F(S(t), I(t))}+\ln \frac{I(t)}{I^{*}} \cdot \frac{F\left(S(t), I^{*}\right)}{F(S(t), I(t))} \leq 0 .
\end{gathered}
$$

Furthermore, by (H7) the following inequality holds:

$$
\left(\frac{I(t)}{I^{*}}-\frac{F(S(t), I(t))}{F\left(S(t), I^{*}\right)}\right)\left(\frac{F\left(S(t), I^{*}\right)}{F(S(t), I(t))}-1\right) \leq 0 .
$$

And by (H8) we have the following inequality:

$$
\left(\frac{G(I(t))}{G\left(I^{*}\right)}-\frac{I(t)}{I^{*}}\right)\left(\frac{I^{*}}{I(t)}-1\right) \leq 0 .
$$

By (29)-(32), we see that $d V_{2} / d t \leq 0$ for all $S(t) \geq 0$, $I(t) \geq 0$. It is easy to verify that the largest invariant set in $\left\{(S(t), I(t)) \mid d V_{2} / d t=0\right\}$ is the singleton $\left\{E^{*}\right\}$. By the Lyapunov-LaSalle asymptotic stability theorem in [31], $E^{*}$ is globally asymptotically stable for any $\tau>0$. This completes the proof.

\section{Discussion and Conclusion}

In this paper, we formulated an SIR epidemic model with delay and two general functions, one is $F(S, I)$ which represents the incidence rate, and the other is $G(I)$ which represents the recovered infected individuals from the infected compartment. We studied the global asymptotic stability of disease-free equilibrium and endemic equilibrium of system (2), respectively. We showed that in Theorem 2 the diseasefree equilibrium $E_{0}=\left(S_{0}, 0\right)$ is locally asymptotically for any $\tau>0$ if the basic reproduction number $R_{0}<1$ and $E_{0}=\left(S_{0}, 0\right)$ are unstable if $R_{0}>1$; in Theorem 3 the diseasefree equilibrium $E_{0}=\left(S_{0}, 0\right)$ is globally asymptotically for any $\tau>0$ if $R_{0} \leq 1$, while in Theorem 4 , the endemic equilibrium $E^{*}=\left(S^{*}, I^{*}\right)$ is globally asymptotically for any $\tau>0$ if $R_{0}>1$.

In order to obtain the global properties of the system (2), we proposed assumptions (H1)-(H8) for functions of $F(S, I)$ and $G(I)$. Conditions (H1)-(H3) are some basic assumptions; for example, (H1) implies that the function $F(S, I)$ is a nonnegative differentiable function on nonnegative quadrant and is positive if and only if both arguments are positive. We used (H4)-(H6), (H7)-(H8) to establish the global asymptotic stability of disease-free equilibrium and endemic equilibrium of system (2), respectively. These hypotheses seem to be mathematical techniques; however, they may be obviously true for many concrete forms of the functions of $F(S, I)$ and $G(I)$ in previous studies.

A special case of system (2) is that when $G(I)=\gamma I, \gamma$ is the recovery rate of the infective individuals. System (2) becomes the following DDEs:

$$
\begin{gathered}
\frac{d S(t)}{d t}=\lambda-\mu S(t)-F(S(t), I(t)), \\
\frac{d I(t)}{d t}=e^{-\mu \tau} F(S(t-\tau), I(t-\tau))-(\mu+\alpha+\gamma) I(t) .
\end{gathered}
$$

The basic reproductive number for system (33) is presented as

$$
R_{0}^{\prime}=\frac{e^{-\mu \tau} F_{I}^{\prime}\left(S_{0}, 0\right)}{\mu+\alpha+\gamma} .
$$

Using Theorems 3 and 4, we can easily obtain the global asymptotic stability of the disease-free equilibrium and the endemic equilibrium of system (33). Regarding to system (33), we now give examples of incidence function $F(S, I)$ that satisfies the required hypotheses obviously.

Example 5. Without delay: let $\tau=0, \lambda=\mu$ and $\delta=\mu+\alpha+\gamma$. Then system (33) becomes to the SIR model studied in [27].

Example 6. Holling type II incidence rate: let $F(S, I)=$ $v_{m} S I /\left(C_{h}+S\right)$ for some constant $v_{m}, C_{h}>0$. Then the hypotheses on $F(S, I)$ are satisfied and the global properties are determined by the basic reproductive number. This model was introduced by $[32,33]$ for considering delays in the standard bacterial growth model in a chemostat. Its global dynamics were first proved by [32] by the fluctuation lemma and a different proof was given in [34, Theorem 5.16] by comparison.

Example 7. Saturate incidence rate: let $F(S, I)=\beta S I /\left(1+\alpha_{1} S+\right.$ $\left.\alpha_{2} I\right)$ for some constant $\alpha_{1}>0, \alpha_{2}>0$. Then hypotheses about $F(S, I)$ are also satisfied and the global properties are determined by the basic reproductive number. The behaviors of this model were previously studied in [23, 24]. In [23], the local stability of disease-free equilibrium and endemic equilibrium was obtained. And in [24], the global stability of disease-free equilibrium and endemic equilibrium was studied.

From these Examples 5-7, we can see that system (2) is reasonably established and it can contain many classical epidemic models and imply their global dynamics as special cases.

\section{Conflict of Interests}

The authors declare that there is no conflict of interests regarding the publication of this paper. 


\section{Acknowledgments}

This work was supported by the National Nature Science Foundation of China (11271303 and 10971168) and the Fundamental Research Funds for the Center Universities (XDJK2009B012).

\section{References}

[1] M. Gabriela, M. Gomes, L. J. White, and G. F. Medley, "The reinfection threshold," Journal of Theoretical Biology, vol. 236, no. 1, pp. 111-113, 2005.

[2] Y. Zhou and H. Liu, "Stability of periodic solutions for an SIS model with pulse vaccination," Mathematical and Computer Modelling, vol. 38, no. 3-4, pp. 299-308, 2003.

[3] A. Gray, D. Greenhalgh, L. Hu, X. Mao, and J. Pan, "A stochastic differential equation SIS epidemic model," SIAM Journal on Applied Mathematics, vol. 71, no. 3, pp. 876-902, 2011.

[4] M. Song, W. Ma, and Y. Takeuchi, "Permanence of a delayed SIR epidemic model with density dependent birth rate," Journal of Computational and Applied Mathematics, vol. 201, no. 2, pp. 389-394, 2007.

[5] W. Ma, M. Song, and Y. Takeuchi, "Global stability of an SIR epidemic model with time delay," Applied Mathematics Letters, vol. 17, no. 10, pp. 1141-1145, 2004.

[6] F. Zhang, Z. Li, and F. Zhang, "Global stability of an SIR epidemic model with constant infectious period," Applied Mathematics and Computation, vol. 199, no. 1, pp. 285-291, 2008.

[7] X. Liu, Y. Takeuchi, and S. Iwami, "SVIR epidemic models with vaccination strategies," Journal of Theoretical Biology, vol. 253, no. 1, pp. 1-11, 2008.

[8] J. Wang, J. Zhang, and Z. Jin, "Analysis of an SIR model with bilinear incidence rate," Nonlinear Analysis, vol. 11, no. 4, pp. 2390-2402, 2010.

[9] A. Korobeinikov and G. C. Wake, "Lyapunov functions and global stability for SIR, SIRS, and SIS epidemiological models," Applied Mathematics Letters, vol. 15, no. 8, pp. 955-960, 2002.

[10] S. M. O’Regan, T. C. Kelly, A. Korobeinikov, M. J. A. O'Callaghan, and A. V. Pokrovskii, "Lyapunov functions for SIR and SIRS epidemic models," Applied Mathematics Letters, vol. 23, no. 4, pp. 446-448, 2010.

[11] L. Liu, X. Q. Zhao, and Y. Zhou, "A tuberculosis model with seasonality," Bulletin of Mathematical Biology, vol. 72, no. 4, pp. 931-952, 2010.

[12] N. Yoshida and T. Hara, "Global stability of a delayed SIR epidemic model with density dependent birth and death rates," Journal of Computational and Applied Mathematics, vol. 201, no. 2, pp. 339-347, 2007.

[13] R. M. Anderson and R. M. May, "Regulation and stability of host-parasite population interactions. I. Regulatory processes," Journal of Animal Ecology, vol. 47, no. 1, pp. 219-267, 1978.

[14] C. Wei and L. Chen, "A delayed epidemic model with pulse vaccination," Discrete Dynamics in Nature and Society, vol. 2008, Article ID 746951, 12 pages, 2008.

[15] J. Zhang, Z. Jin, Q. Liu, and Z. Zhang, "Analysis of a delayed SIR model with nonlinear incidence rate," Discrete Dynamics in Nature and Society, vol. 2008, Article ID 636153, 16 pages, 2008.

[16] Z. Jiang and J. Wei, "Stability and bifurcation analysis in a delayed SIR model," Chaos, Solitons and Fractals, vol. 35, no. 3, pp. 609-619, 2008.
[17] R. Xu and Z. Ma, "Stability of a delayed SIRS epidemic model with a nonlinear incidence rate," Chaos, Solitons and Fractals, vol. 41, no. 5, pp. 2319-2325, 2009.

[18] R. Xu, Z. Ma, and Z. Wang, "Global stability of a delayed SIRS epidemic model with saturation incidence and temporary immunity," Computers \& Mathematics with Applications, vol. 59, no. 9, pp. 3211-3221, 2010.

[19] R. Xu and Z. Ma, "Global stability of a SIR epidemic model with nonlinear incidence rate and time delay," Nonlinear Analysis, vol. 10, no. 5, pp. 3175-3189, 2009.

[20] X. Zhang and X. Liu, "Backward bifurcation of an epidemic model with saturated treatment function," Journal of Mathematical Analysis and Applications, vol. 348, no. 1, pp. 433-443, 2008.

[21] C. C. McCluskey, "Global stability for an SIR epidemic model with delay and nonlinear incidence," Nonlinear Analysis, vol. 11, no. 4, pp. 3106-3109, 2010.

[22] A. Kaddar, "On the dynamics of a delayed SIR epidemic model with a modified saturated incidence rate," Electronic Journal of Differential Equations, vol. 2009, no. 133, pp. 1-7, 2009.

[23] A. Kaddar, A. Abta, and H. T. Alaoui, "A comparison of delayed SIR and SEIR epidemic models," Nonlinear Analysis, vol. 16, no. 2, pp. 181-190, 2011.

[24] A. Abta, A. Kaddar, and H. T. Alaoui, "Global stability for delay SIR and SEIR epidemic models with saturated incidence rates," Electronic Journal of Differential Equations, vol. 2012, no. 23, pp. $1-13,2012$.

[25] Z. Liu, "Dynamics of positive solutions to SIR and SEIR epidemic models with saturated incidence rates," Nonlinear Analysis, vol. 14, no. 3, pp. 1286-1299, 2013.

[26] A. Korobeinikov and K. Philip Maini, "Nonliear incidence and stability of infectious diseasemodels," Mathematical Medicine and Biology, vol. 22, pp. 113-128, 2005.

[27] A. Korobeinikov, "Lyapunov functions and global stability for SIR and SIRS epidemiological models with non-linear transmission," Bulletin of Mathematical Biology, vol. 68, no. 3, pp. 615-626, 2006.

[28] A. Korobeinikov, "Global properties of infectious disease models with nonlinear incidence," Bulletin of Mathematical Biology, vol. 69, no. 6, pp. 1871-1886, 2007.

[29] A. Korobeinikov, "Stability of ecosystem: global properties of a general predator-prey model," Mathematical Medicine and Biology, vol. 26, no. 4, pp. 309-321, 2009.

[30] G. Huang, Y. Takeuchi, W. Ma, and D. Wei, "Global stability for delay SIR and SEIR epidemic models with nonlinear incidence rate," Bulletin of Mathematical Biology, vol. 72, no. 5, pp. 11921207, 2010.

[31] J. K. Hale and S. M. Verduyn Lunel, Introduction to Functional Differential Equations, Springer, New York, NY, USA, 1993.

[32] S. F. Ellermeyer, "Competition in the chemostat: global asymptotic behavior of a model with delayed response in growth," SIAM Journal on Applied Mathematics, vol. 54, no. 2, pp. 456465, 1994.

[33] S. F. Ellermeyer, J. Hendrix, and N. Ghoochan, "A theoretical and empirical investigation of delayed growth response in the continuous culture of bacteria," Journal of Theoretical Biology, vol. 222, no. 4, pp. 485-494, 2003.

[34] H. Smith, An Introduction to Delay Differential Equations with Applications to the Life Sciences, Springer, New York, NY, USA, 2011. 


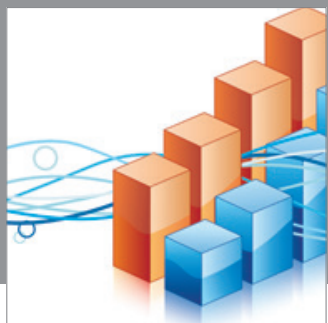

Advances in

Operations Research

mansans

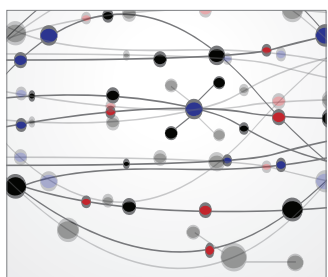

The Scientific World Journal
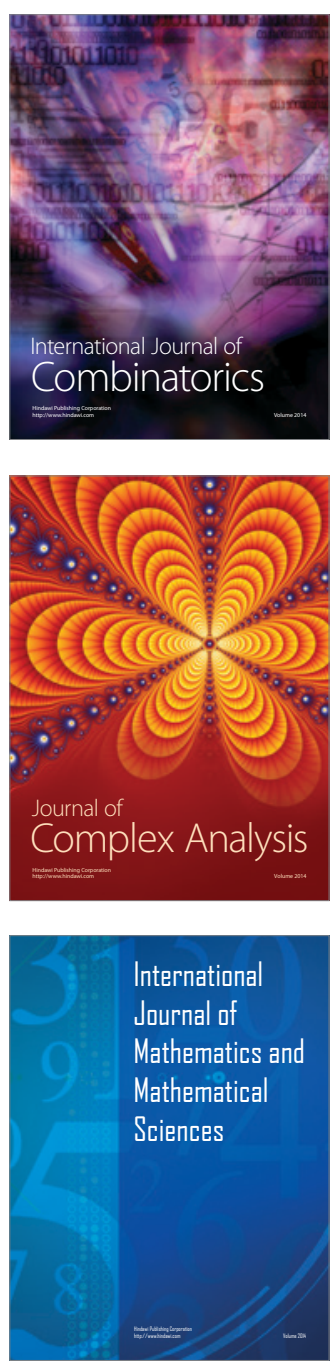
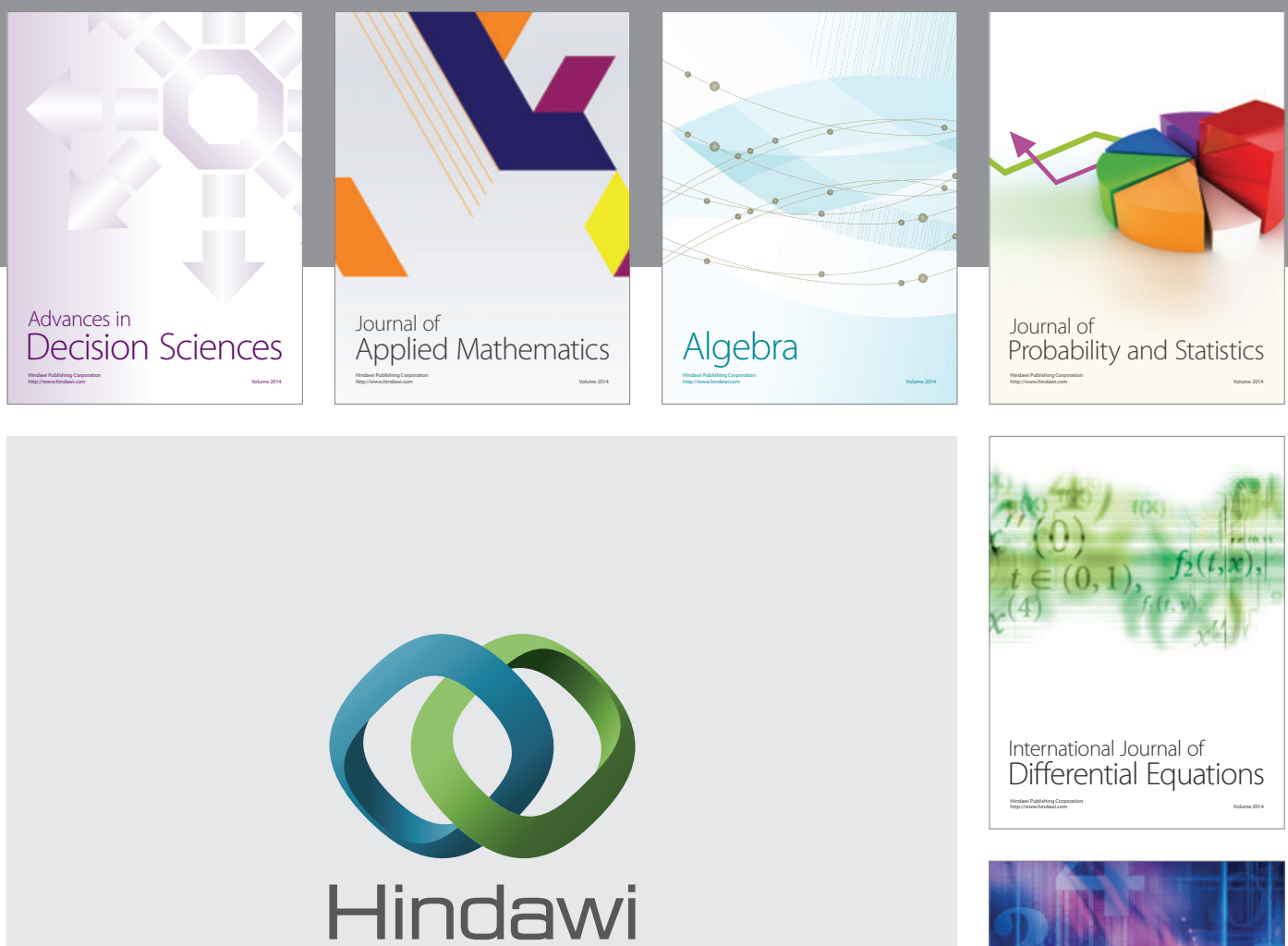

Submit your manuscripts at http://www.hindawi.com
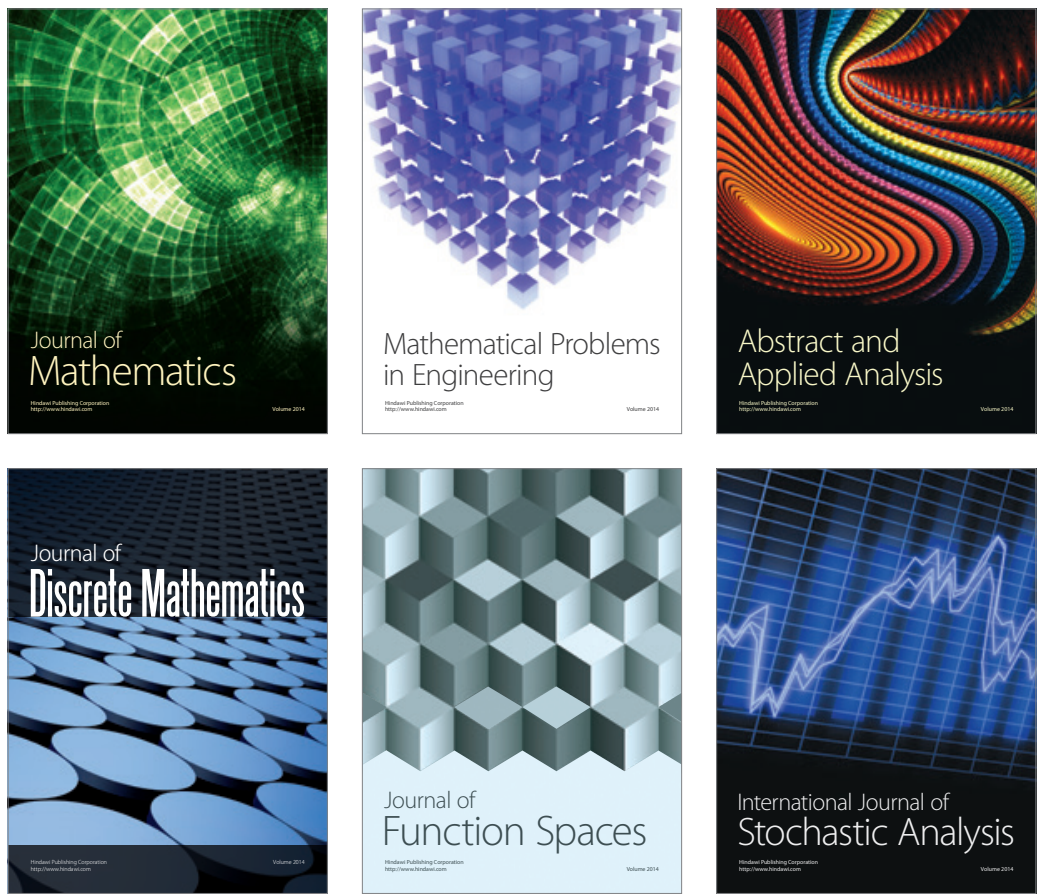

Journal of

Function Spaces

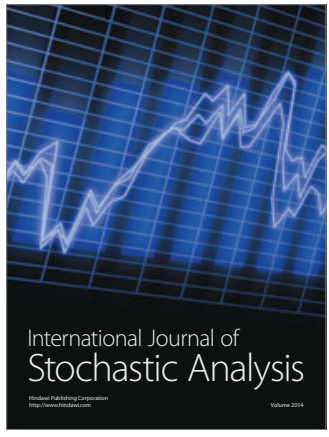

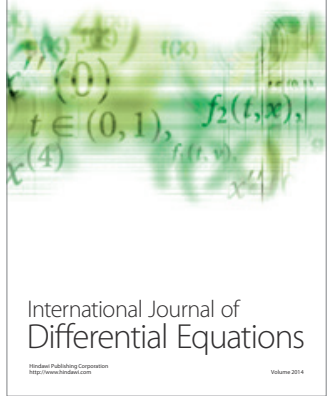
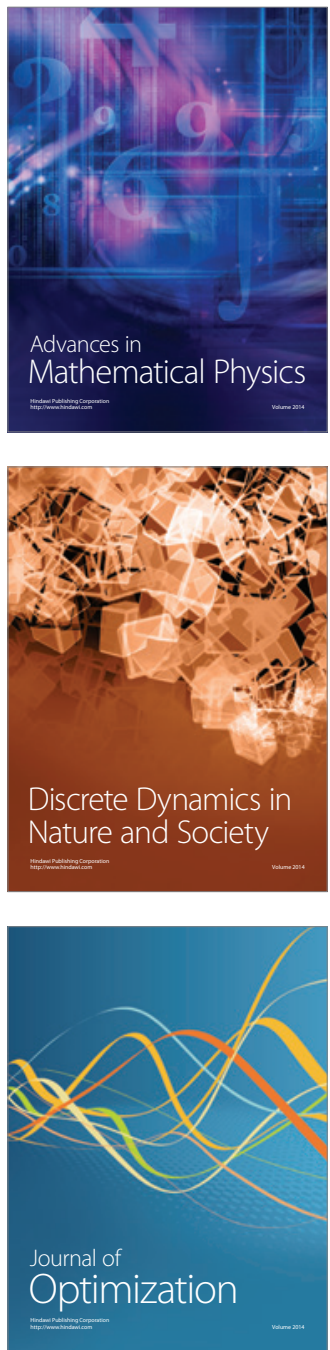